\title{
Rice Cookie Decreases Plasma and Hepatic Lipid Levels in High-Fat Diet-fed Mice: A Comparison Study with Traditional Western Style Cookies
}

\author{
Sun Hee Hong, Mijeong Kim, Minji Woo, Yeong Ok Song* \\ Department of Food Science and Nutrition, Kimchi Research Institute, Pusan National University, Busan 46241, Republic of Korea \\ *Corresponding author: yosong@pusan.ac.kr
}

\begin{abstract}
The lipid-lowering and antioxidant effects of the traditional Korean rice cookie (KRC), dasik, were compared with those of a western style cookie (WSC) in mice fed a high-fat diet (HFD). The KRC or WSC was supplemented to the HFD as $7 \%$ of the total calories. The experimental groups $(n=7)$ were the normal diet group, HFD group, HFD-KRC group, and HFD-WSC group. The plasma and hepatic triglyceride concentrations of the HFD-KRC group were found to be lower than those of the HFD-WSC group as a result of sterol regulatory element-binding protein 1 and fatty acid synthase expression downregulation, and concomitant peroxisome proliferator-activated receptor-alpha, carnitine palmitoyltransferase 1 , and acyl-coenzyme A oxidase 1 expression upregulation $(\mathrm{p}<0.05)$. The hepatic reactive oxygen species and peroxynitrite levels were also diminished in the HFD-KRC group of mice, whereas their catalase and glutathione peroxidase protein expression levels were higher than those in the HFD and HFD-WSC groups $(p<0.05)$. In conclusion, the lipid-lowering effects and antioxidant property of the KRC were greater than those of the WSC in mice fed a HFD. Thus, the choice of KRCs as a snack would be preferable to choosing WSCs.
\end{abstract}

Keywords: rice cookie, high-fat diet, lipid, antioxidant, transcription factor

Cite This Article: Sun Hee Hong, Mijeong Kim, Minji Woo, and Yeong Ok Song, "Rice Cookie Decreases Plasma and Hepatic Lipid Levels in High-Fat Diet-fed Mice: A Comparison Study with Traditional Western Style Cookies.” Journal of Food and Nutrition Research, vol. 5, no. 7 (2017): 451-457. doi: 10.12691/jfnr-5-7-1.

\section{Introduction}

Calorie-heavy snacks have become a major concern in regard to public health. In the USA, calories from snacking have increased steadily to approximately 25\% of the daily calorie intake [1]. Moreover, the nutritional value of traditional western style cookies (WSCs), made using refined flour, butter, eggs, and sugar, is considered to be poor because of their high sugar and fat, but low fiber, vitamin, and mineral, contents [2]. It is a well-known fact that these ingredients, particularly fat and sugar, are positively associated with plasma lipid elevation [3]. In contrast, the Korean traditional rice cookie (KRC), dasik (meaning "a tea food”), is made from steamed rice-cake flour, mung bean starch, and honey. Other sugar and fat sources are not used and further baking or frying is not required for dasik preparation.

Steamed rice has shown hypolipidemic effects in hamsters fed a high-fat diet (HFD), by downregulating the expression of transcription factors and genes related to cholesterol and fatty acid synthesis and upregulating those related to lipid oxidation [4]. The glycemic index (GI) of mung bean starch is low, which is advantageous since diets with a low GI have revealed serum lipid-lowering effects [5]. Therefore, these carbohydrate sources of
KRCs might have a more beneficial effect on plasma lipid profiles than the wheat flour of WSCs. Aside from the carbohydrate source; the type of sweetener used for cookie preparation might also change the plasma lipid concentration. Sugar, the main sweetener in WSCs, is cited as a positive attributor of hyperlipidemia [6], whereas honey has exerted triglyceride (TG)- and total cholesterol (TC)-lowering effects in humans [7].

Hyperlipidemia, a condition characterized by a high level of TG or cholesterol in the blood [8], is a reflection of increased fatty acid synthesis and decreased hepatic lipid oxidation. The biosyntheses of cholesterols, fatty acids, and TGs are tightly regulated by a family of transcription factors named sterol regulatory element-binding proteins (SREBPs) [9]. SREBP-1 mainly stimulates fatty acid synthesis via the upregulation of acetyl-CoA carboxylase (ACC) and fatty acid synthase (FAS). Transcription factor SREBP-2 regulates the enzymes involved in cholesterol biosynthesis, including 3-hydroxy-3-methylglutaryl-CoA reductase (HMGCR) [10]. Patients with hyperlipidemia are usually treated with a drug or functional food that inhibits SREBP expression [10]. Lipid catabolism, in particular fatty acid oxidation, is principally regulated by peroxisome proliferator-activated receptor-alpha (PPAR- $\alpha$ ) through the upregulation of enzymes such as carnitine palmitoyltransferase 1 (CPT1) and acyl-coenzyme A oxidase 1 (ACOX1) [11]. The major route for plasma 
cholesterol elimination is via bile acid secretion. The microsomal cytochrome P450 family 7 subfamily A member 1 (CYP7A1; also known as cytochrome P450 cholesterol 7 alpha-hydroxylase or cytochrome P450 cholesterol 7 alpha-monooxygenase) is the rate-limiting enzyme that catalyzes the conversion of cholesterol into bile acids in the liver [12].

Under the condition of hyperlipidemia, oxidative stress is commonly observed. Reactive oxygen species (ROS) are produced in living organisms as a result of normal cellular metabolism and environmental factors [13]. Aerobic organisms have integrated antioxidant systems (including enzymatic and non-enzymatic antioxidants) that prevent the oxidative damage from ROS [13]. However, oxidative stress is elevated if the antioxidant systems are not properly activated, which can lead to the development of chronic diseases such as atherosclerosis, hypertension, cancer, neurological disorders, diabetes, and acute respiratory distress syndrome $[13,14]$. Postprandial oxidative stress is characterized by an increased susceptibility of organisms toward oxidative damage after consumption of a meal rich in lipids and/or carbohydrates [15]. Thus, the macronutrients in foods can have effects on the redox balance in the body, where they either are a target of oxidative modifications after absorption or are present in a prooxidant form in the diet. Hyperlipidemia and hyperglycemia are cited as positive contributors to elevated oxidative damage, through their effects on lipoprotein levels and the antioxidant status [16]. Postprandial increases of plasma lipid and carbohydrate levels therefore increase the risk of atherosclerosis and related disorders by increasing oxidative stress [17].

In this study, the health benefits of the KRC and of a traditional WSC were compared by evaluating their effects on the plasma and hepatic lipid concentrations, ROS production, lipid peroxidation, and antioxidant status (and its related mechanisms) in HFD-fed mice.

\section{Materials and Methods}

\subsection{Preparation of the Cookie Samples}

The KRC was made up of 55.6\% rice cake flour, $22.2 \%$ mung bean starch, and 22.2\% honey. The ingredients were mixed thoroughly and an exact amount of the dough was then placed into a special-purpose mold. Commercially available WSCs (Lotus Bakeries, Kaprijke, Belgium) were purchased at a local market, and according to the manufacturer's information, the ingredients were wheat flour, sugar, vegetable oils, brown sugar, sodium bicarbonate, soy flour, salt, and cinnamon.

\subsection{Evaluation of the Nutritional Value of the Cookie Samples}

The nutritional value of both types of cookies, including calories and contents of protein, fat, carbohydrate, and fiber, were determined using a diet-analyzing program (CAN-pro 3.0; Korean Nutrition Society, Seoul, Korea). For the KRC, the recipe was used. For the WSC, the nutrition information on the product label was used. The nutritional value was expressed as $\mathrm{kcal} / 100 \mathrm{~g}$ or $\mathrm{g} / 100 \mathrm{~g}$.

\subsection{Experimental Diets and Animals}

As shown in Table 1, an AIN-76 synthetic diet, a HFD, and a HFD containing KRC or WSC were prepared. The HFD was prepared by adding lard and cholesterol to the AIN-76 diet to provide $46.53 \%$ of the total calories from fats. For the dessert diet, the KRC or WSC was supplemented to the HFD as $7 \%$ of the total calories, which were calculated as follows; calories from daily snack consumption has been reported approximately $25 \%$ to total calorie intake and calorie from carbohydrate-dessert is accounted as $26 \%$ to total calorie from all snacks [1] (25\% * $0.26 \fallingdotseq$ $7 \%)$. The normal diet contained $3.9 \mathrm{kcal} / \mathrm{g}$, whereas the HFD, HFD-KRC, and HFD-WSC contained $5.0 \mathrm{kcal} / \mathrm{g}$.

Table 1. Composition of the experimental diets

\begin{tabular}{lcccc}
\hline \multirow{2}{*}{ Ingredient (g) } & ND $^{1)}$ & HFD $^{2)}$ & \multicolumn{2}{c}{ HFD + dessert } \\
\cline { 3 - 5 } & & & KRC & WSC \\
\hline Casein & 200 & 171 & 175 & 176 \\
DL-Methionine & 3 & 3 & 3 & 3 \\
Corn starch & 350 & 203 & 127 & 128 \\
Sucrose & 300 & 279 & 262 & 264 \\
Cellulose & 50 & 43 & 44 & 44 \\
Corn oil & 50 & 39 & 41 & 41 \\
Lard & & 217 & 222 & 224 \\
Cholesterol & 55 & 30 & 5 & 5 \\
Mineral mix & 10 & 9 & 31 & 31 \\
Vitamin mix & 2 & 2 & 9 & 9 \\
Choline bitartrate & & & 2 & 2 \\
\hline Cookie & & & 59 & 73 \\
Rice cake flour & & & 22 & \\
Mung bean starch & & & 24 & \\
Brown rice flour & & & & \\
Honey & & & & \\
\hline
\end{tabular}

HFD, high-fat diet; KRC, Korean rice cookie; WSC, Western style cookie.

${ }^{1)}$ Normal diet (ND) was prepared synthetically according to AIN-76A guidelines.

${ }^{2)}$ The calories from fat in the HFD was $46.53 \%$ of the total calories.

${ }^{3)}$ The calories from the KRC or WSC in the HFD was $7 \%$ of the total calories.

Male ICR mice ( $n=28 ; 4$ weeks old) were purchased from Orient Bio Inc. (Seongnam, Korea). The animals were kept in individual cages during the entire experimental period under the controlled condition of $23 \pm 1{ }^{\circ} \mathrm{C}$ and $50 \%$ humidity with a 12 h light:dark cycle. After 1 week of acclimatization, the mice were randomly assigned into the four experimental groups: normal diet (NOR) group, HFD group, KRC-containing HFD (HFD-KRC) group, and WSC-containing HFD (HFD-WSC) group. The animals had free access to the diet and water for 9 weeks. The food consumption and body weight of the mice were measured every week. The animal protocol was reviewed for ethical procedures and scientific care and approved by the Pusan National University-Institutional Animal Care and Use Committee (Approval No. PNU-2012-0118).

\subsection{Plasma, Liver, and Epididymal Fat Pad Collection}

After $12 \mathrm{~h}$ of fasting, the mice were anesthetized by intraperitoneal administration of $30 \mathrm{mg} / \mathrm{kg}$ of a zolazepam and tiletamine combination (Zoletil 50; Virbac Laboratories, 
Carros, France) and $10 \mathrm{mg} / \mathrm{kg}$ of xylazine (Rompun; Bayer Korea, Seoul, Korea). Blood was collected from the inferior vena cava into heparin tubes, which were then centrifuged at $3,012 \times g$ and $4^{\circ} \mathrm{C}$ for 20 min to obtain the plasma. The liver was removed after perfusion with ice-cold phosphate-buffered saline (PBS), and after impurities had been detached, it was rinsed several times with PBS and then weighed. Epididymal fat pads were excised and weighed. The plasma and liver samples were placed immediately on dry ice and stored at $-80^{\circ} \mathrm{C}$ for further analysis.

\subsection{Determination of Biochemical Parameters in the Plasma and Liver}

The plasma leptin concentration was measured using a leptin ELISA kit (Cat. No. KMC2281; Invitrogen Co., Camarillo, CA, USA) according to the manufacturer's protocol. The detection sensitivity of the assay is $<50$ $\mathrm{pg} / \mathrm{mL}$. Plasma alanine transaminase (ALT) and aspartate transaminase (AST) activities were measured with a commercially available kit (AM101-K; Asan Pharm., Seoul, Korea)

Plasma TG and TC concentrations were measured using commercial kits (AM157S-K and AM202-K, respectively; Asan Pharm.). The hepatic lipids were extracted from the liver tissue using a modified method of Folch et al. [18]. Hepatic TG and TC concentrations were measured with the same kits used for the plasma lipid analyses.

\subsection{Determination of Hepatic Oxidative Stress-related Parameters}

Liver homogenates used for thiobarbituric acid-related substances (TBARS) and glutathione (GSH) concentration determination were prepared by homogenizing hepatic tissue with ice-cold PBS (1:9, w/w). The post mitochondrial fraction of liver used for determining the ROS and peroxynitrite $\left(\mathrm{ONOO}^{-}\right)$levels was obtained by centrifugation of the liver homogenates at $18,627 \times g$ and $4^{\circ} \mathrm{C}$ for $20 \mathrm{~min}$. Hepatic lipid peroxidation was determined as TBARS and expressed as the malondialdehyde (MDA) concentration [19]. The hepatic GSH concentration was measured using the method of Ellman [20]. Hepatic ROS and ONOO levels were measured by $2^{\prime}, 7^{\prime}$-dichlorofluorescein diacetate and dihydrorhodamine 123 assays [21], respectively. Changes in fluorescence of the reaction mixture were measured for $30 \mathrm{~min}$ at an excitation wavelength of 485 $\mathrm{nm}$ and an emission wavelength of $530 \mathrm{~nm}$.

\subsection{Western Blot Analysis}

To obtain a whole-cell hepatic fraction, liver tissue was homogenized in ice-cold lysis buffer (50 mM Tris, pH 8.0, $5 \mathrm{mM}$ EDTA, $150 \mathrm{mM} \mathrm{NaCl}$, and 1\% nonidet-P40 containing protease inhibitor cocktail) using a Polytron homogenizer (PT-MR 3100; Polytron, Kinematica, Lucerne, Switzerland), placed on ice for $1 \mathrm{~h}$, and then centrifuged at $18,627 \times g$ and $4^{\circ} \mathrm{C}$ for $20 \mathrm{~min}$. Sodium dodecyl sulfate polyacrylamide gel electrophoresis of the fraction was performed using a previously reported method [22], in preparation for western blot analysis. The primary antibodies used for the western blot assay were those for SREBP-1 (H-160; sc-8984), ACC $\alpha$ (T-18; sc-26817), PPAR $\alpha$ (H-98; sc-9000), CPT1-C (S-17; sc-139482), ACOX1 (H-140; sc-98499), SREBP-2 (H-164; sc-5603), HMGCR (H-300; sc-33827), CYP7A1 (H-58; sc-25536), SOD-1 (FL-154; sc-11407), catalase (F-17; sc-34285), and glutathione peroxidase (GPx-1/2) (B-6; sc-133160) from Santa Cruz Biotechnology Inc. (Santa Cruz, CA, USA), and anti-fatty acid synthase (ab22759) from Abcam Inc. (Cambridge, UK). The horseradish peroxidase-conjugated secondary antibodies used (all procured from Abcam Inc.) were rabbit polyclonal secondary antibody to mouse IgG-H\&L (ab6728), donkey polyclonal secondary antibody to rabbit IgG-H\&L (ab6802), and rabbit polyclonal secondary antibody to goat IgG-H\&L (ab6741). Protein expression was visualized by enhanced chemiluminescence-based detection using the CAS-400 imaging system (Core Bio, Seoul, Korea). The band densities were measured using ImageJ software (National Institutes of Health, Bethesda, MD, USA), where corresponding protein amounts were normalized to the value of $\beta$-actin.

\subsection{Statistical Analysis}

Data are presented as the mean \pm standard deviation. Statistical significance of differences in the mean values among the four groups was assessed by one-way analysis of variance, followed by Duncan's multiple-range test as a post hoc analysis to determine where the significance exists at $\mathrm{p}<0.05$. Student's $t$-test was used to determine differences between two groups.

\section{Results and Discussion}

\subsection{Nutritional Evaluation of the Two Types of Cookies}

In $100 \mathrm{~g}$ of the KRC versus $100 \mathrm{~g}$ of the WSC, the calorie amount was $347.9 \mathrm{kcal}$ versus $496.0 \mathrm{kcal}$, the carbohydrate content was $75.1 \mathrm{~g}$ versus $73.3 \mathrm{~g}$, the protein content was $8.0 \mathrm{~g}$ versus $6.7 \mathrm{~g}$, and the fat content was $1 \mathrm{~g}$ versus $20 \mathrm{~g}$, respectively.

\subsection{Body Weight, Epididymal Fat Mass, and Leptin Level}

As shown in Table 2, the body weight gain, epididymal fat mass, and leptin level of the HFD-KRC group were significantly lower than those of the HFD-WSC group $(p<0.05)$. This might be due to the different ingredients used in each type of cookie, even though the calories provided to the experimental groups were the same. Although the carbohydrate contents in the KRC and WSC were the same, the grain source for the $\mathrm{KRC}$ is rice and that for the WSC is wheat. As mentioned before, cooked rice was reported to lower the epididymal and retroperitoneal fat mass of hamsters fed a HFD [4]. In addition, the lipid content in the WSC was 20-fold higher than that in the KRC (20 g/100 g vs $1 \mathrm{~g} / 100 \mathrm{~g})$. The lack of fiber in the WSC might have attributed to the body weight gain in the HFD-WSC group. 
Table 2. Food intake, body weight gain, epididymal fat mass, and leptin level in mice fed a cookie-supplemented high-fat diet for 9 weeks

\begin{tabular}{lccc}
\hline Group $^{1)}$ & Food intake $(\mathrm{g})$ & Body weight gain $(\mathrm{g})$ & Epididymal fat mass $(\mathrm{g} / 100 \mathrm{~g} \mathrm{bw})$ \\
\hline NOR & $4.53 \pm 0.14^{\mathrm{NS}}$ & $7.95 \pm 1.16^{\mathrm{c}}$ & $0.90 \pm 0.24^{\mathrm{c}}$ \\
HFD & $4.51 \pm 0.17$ & $20.10 \pm 0.60^{\mathrm{a}}$ & $3.95 \pm 0.24^{\mathrm{a}}$ \\
HFD-KRC & $4.56 \pm 0.45$ & $15.05 \pm 1.68^{\mathrm{b}}$ & $2.98 \pm 0.54^{\mathrm{b}}$ \\
HFD-WSC & $4.67 \pm 0.24$ & $20.50 \pm 3.85^{\mathrm{a}}$ & $3.80 \pm 0.85^{\mathrm{a}}$ \\
\hline
\end{tabular}

Data are the mean \pm SD ( $=7$ per group)

${ }^{1)}$ NOR, mice fed the AIN-76 diet; HFD, mice fed the high-fat diet (HFD) only; HFD-KRC, mice fed the HFD supplemented with the Korean rice cookie (KRC); HFD-WSC, mice fed the HFD supplemented with the Western style cookie (WSC).

${ }^{\mathrm{a}-\mathrm{c}}$ Data with different letters in the column are significantly different according to one-way ANOVA followed by Duncan's multiple range test at $\mathrm{p}<0.05$.

${ }^{\mathrm{NS}}$ Not significantly different at $\mathrm{p}<0.05$.

A HFD increases the body weight and results in adipose tissue accumulation [23]. Leptin, a hormone secreted primarily by adipocytes, controls the body weight by regulating food intake and increasing energy expenditure [24]. Leptin levels are positively correlated with body weight and epididymal fat mass [25].

\subsection{Lipid-lowering Effects of Korean Rice Cookie}

Compared with the levels in the NOR group, the plasma TG and TC concentrations of the HFD group had increased by $151.96 \%$ and $151.39 \%$, respectively $(\mathrm{p}<0.05$, Table 3), and the hepatic concentrations were likewise augmented by $474 \%$ and $609 \%$, respectively $(\mathrm{p}<0.05$, Table 3). These results are in line with other studies that showed a significant increase in plasma and hepatic lipid levels induced by a HFD [26]. Among the HFD groups, the plasma and hepatic TG concentrations of the HFD-KRC group were significantly lower than those of the HFD group $(\mathrm{p}<0.05)$, but no differences were found in the plasma and hepatic TC levels. However, when the plasma and hepatic lipid levels were compared between the HFD-KRC and HFD-WSC groups, significant differences were observed ( $\mathrm{p}<0.05$ by Student's $t$-test). In particular, the plasma and hepatic TG concentrations of the HFD-KRC group were significantly lower (by 23.35\% and $21.36 \%$, respectively, $\mathrm{p}<0.05$ ) than those of the HFD-WSC group. Since the lipid content in the WSC was 20-fold higher than that in the KRC, the lipid-lowering effect of the KRC might be due to its lower lipid content as well as its carbohydrate sources, which were different from those of the WSC. A diet supplemented with cooked rice decreased the plasma and hepatic lipid concentrations in HFD-fed hamsters [4]. Moreover, honey was demonstrated to have plasma TG- and TC-lowering effects in humans [27], whereas sugar is a known attributor of hyperlipidemia [28]. Mung bean starch with its low GI might be another contributor of the lower lipid concentration, as diets with a low GI have demonstrated serum lipid-lowering effects [5].

\subsection{Inhibition of Hepatic Triglyceride Metabolism by Korean Rice Cookie}

Protein expression of the mature forms of SREBP-1, ACC, and FAS in the HFD group was increased by $185.48 \%$, $168.42 \%$, and $174.07 \%$, respectively, relative to the levels in the NOR group $(\mathrm{p}<0.05$; Figure 1$)$, which were in good agreement with the hepatic TG results observed in this study. The expression levels of the lipogenic transcription factor and enzymes were similar between the HFD-WSC and HFD groups, but were comparatively lower in the HFD-KRC group $(\mathrm{p}<0.05)$. In particular, the SREBP-1 and FAS expression levels of the HFD-KRC group were significantly decreased by $35.00 \%$ and $31.87 \%$, respectively, compared with those of the HFD-WSC group. In the case of lipid oxidation factors, the expression levels of PPAR $\alpha, \mathrm{CPT} 1$, and ACOX1 were lower in the HFD group than in the NOR group $(\mathrm{p}<0.05)$. Among the HFD-fed groups, the HFD-KRC group had higher expression of transcription factor and enzymes related to fatty acid oxidation than the HFD and HFD-WSC groups $(\mathrm{p}<0.05)$. In the HFD-KRC group, the expression of PPAR $\alpha, \mathrm{CPT} 1$, and ACOX1 was increased by $131.09 \%, 175.20 \%$, and $202.18 \%$, respectively, compared with the HFD group, and by $154.32 \%, 130.18 \%$, and $154.64 \%$, respectively, compared with the HFD-WSC group. Since cooked rice has been reported to reduce the expression of lipogenic genes (e.g., SREBP-1, ACC, and FAS), while increasing the $\beta$-oxidation-related genes (including PPAR $\alpha$ and its target enzymes CPT1 and ACOX1) in HFD-fed hamster [4], the lipid-lowering effects of the KRC in our study are likely due to its major ingredient, rice cake flour.

\subsection{Effects of Korean Rice Cookie on the Regulation of Hepatic Cholesterol Metabolism}

SREBP-2 regulates the TC level in the body by increasing cholesterol biosynthetic enzymes, including HMGCR [10], whereas CYP7A1 catalyzes the excretion of cholesterol via the digestive tract [12]. In this study, the mature forms of SREBP-2 and HMGCR were increased and CYP7A1 was decreased in the HFD group relative to the levels in the NOR group $(\mathrm{p}<0.05$, Figure 2$)$. HFD-KRC significantly decreased the expression of SREBP-2 and HMGCR and increased that of CYP7A1 relative to the levels in the HFD group $(\mathrm{p}<0.05)$. In addition, SREBP-2 and HMGCR expressions were $20.69 \%$ and $21.32 \%$ lower, respectively, in the HFD-KRC group than in the HFD-WSC group $(\mathrm{p}<0.05)$. In the same way that cooked rice lowered cholesterol levels via the regulation of cholesterol metabolism, downregulation of HMGCR mRNA and protein levels, and upregulation of CYP7A1 expression in HFD-fed hamsters [4], so too had the rice flour in the KRC executed similar effects in HFD-fed mice. 
Table 3. Biochemical parameters in the plasma and liver of mice fed a cookie-supplemented high-fat diet for 9 weeks

\begin{tabular}{|c|c|c|c|c|}
\hline Group $^{1)}$ & NOR & HFD & HFD-KRC & HFD-WSC \\
\hline \multicolumn{5}{|l|}{ Plasma } \\
\hline AST & $57.30 \pm 5.77^{\mathrm{b}}$ & $74.50 \pm 8.85^{\mathrm{a}}$ & $60.80 \pm 2.87^{\mathrm{b}}$ & $59.19 \pm 9.66^{b}$ \\
\hline ALT & $49.47 \pm 4.68^{\mathrm{b}}$ & $63.38 \pm 7.14^{\mathrm{a}}$ & $52.31 \pm 2.33^{\mathrm{b}}$ & $51.00 \pm 7.82^{\mathrm{b}}$ \\
\hline TG & $28.52 \pm 1.30^{\mathrm{b}}$ & $43.34 \pm 4.45^{\mathrm{a}}$ & $35.33 \pm 4.67^{\mathrm{b}}$ & $46.09 \pm 6.83^{\mathrm{a}}$ \\
\hline TC & $102.53 \pm 29.21^{b}$ & $155.22 \pm 16.58^{\mathrm{a}}$ & $132.23 \pm 12.94^{\mathrm{a}, *}$ & $136.23 \pm 22.45^{\mathrm{a}}$ \\
\hline \multicolumn{5}{|l|}{ Liver } \\
\hline TG & $21.06 \pm 4.81^{\mathrm{d}}$ & $99.82 \pm 13.95^{\mathrm{a}}$ & $61.66 \pm 14.58^{\mathrm{c}}$ & $78.41 \pm 9.48^{\mathrm{b}}$ \\
\hline TC & $2.42 \pm 0.33^{c}$ & $14.73 \pm 3.20^{\mathrm{a}}$ & $10.82 \pm 1.49^{\mathrm{b}}$ & $12.60 \pm 0.74^{\mathrm{ab}}$ \\
\hline ROS & $1.00 \pm 0.12^{\mathrm{c}}$ & $2.29 \pm 0.88^{\mathrm{ab}}$ & $1.68 \pm 0.36^{\mathrm{bc}}$ & $2.54 \pm 0.76^{\mathrm{a}}$ \\
\hline $\mathrm{ONOO}^{-}$ & $1.00 \pm 0.44^{c}$ & $2.06 \pm 0.72^{\mathrm{ab}}$ & $1.34 \pm 0.41^{\mathrm{bc}}$ & $2.88 \pm 0.91^{\mathrm{a}}$ \\
\hline TBARS & $6.20 \pm 0.10^{\mathrm{b}}$ & $10.59 \pm 2.23^{\mathrm{a}}$ & $6.34 \pm 1.18^{\mathrm{b}}$ & $8.14 \pm 2.48^{\mathrm{ab}}$ \\
\hline GSH & $0.090 \pm 0.066^{\mathrm{a}}$ & $0.030 \pm 0.016^{\mathrm{b}}$ & $0.069 \pm 0.030^{\mathrm{ab},{ }^{*}, \dagger}$ & $0.028 \pm 0.014^{\mathrm{b}}$ \\
\hline
\end{tabular}

Data are the mean \pm SD ( $n=7$ per group).

ALT, alanine transaminase; AST, aspartic acid transaminase; GSH, glutathione; ONOO-, peroxynitrite; ROS, reactive oxygen species; TBARS, thiobarbituric acid-related substances; TC, total cholesterol; TG, triglycerides.

${ }^{1)}$ NOR, mice fed the AIN-76 diet; HFD, mice fed the high-fat diet only; HFD-KRC, mice fed the HFD supplemented with the Korean rice cookie (KRC); HFD-WSC, mice fed the HFD supplemented with the Western style cookie (WSC).

${ }^{\mathrm{a}-\mathrm{d}}$ Data with different letters in the row are significantly different according to one-way ANOVA followed by Duncan's multiple range test at $\mathrm{p}<0.05$.

*Significantly different according to Student's $t$-test at $\mathrm{p}<0.05$ compared with the HFD group.

tSignificantly different according to Student's $t$-test at $\mathrm{p}<0.05$ compared with the HFD-WSC group.

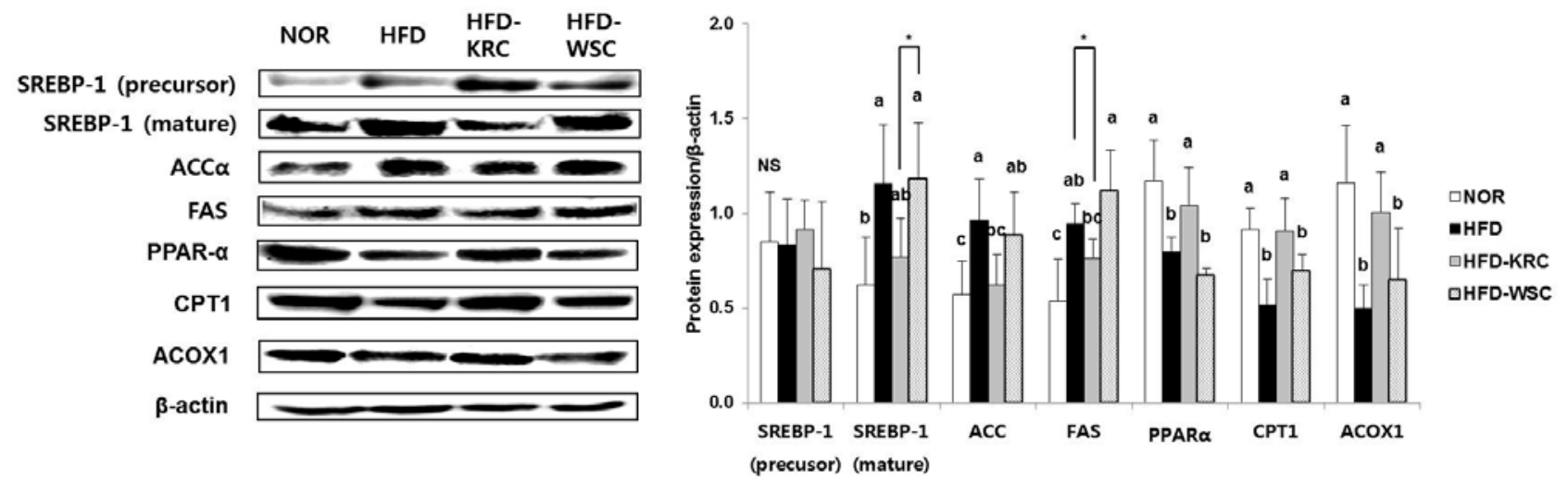

Figure 1. Protein expression of transcription factors and enzymes involved in fatty acid metabolism in the liver of mice fed a cookie-supplemented high-fat diet for 9 weeks. NOR, mice fed the AIN-76 diet; HFD, mice fed the high fat diet only; HFD-KRC, mice fed the HFD supplemented with the Korean rice cookie; HFD-WSC, mice fed the HFD supplemented with the Western style cookie. ${ }^{\text {a-c } D a t a ~ w i t h ~ d i f f e r e n t ~ l e t t e r s ~ a r e ~ s i g n i f i c a n t l y ~ d i f f e r e n t ~}$ according to one-way ANOVA followed by Duncan's multiple range test at $\mathrm{p}<0.05$. "Significantly different according to Student's $t$-test at $\mathrm{p}<0.05$ between two groups. ${ }^{\text {NS }}$ Not significantly different at $\mathrm{p}<0.05$

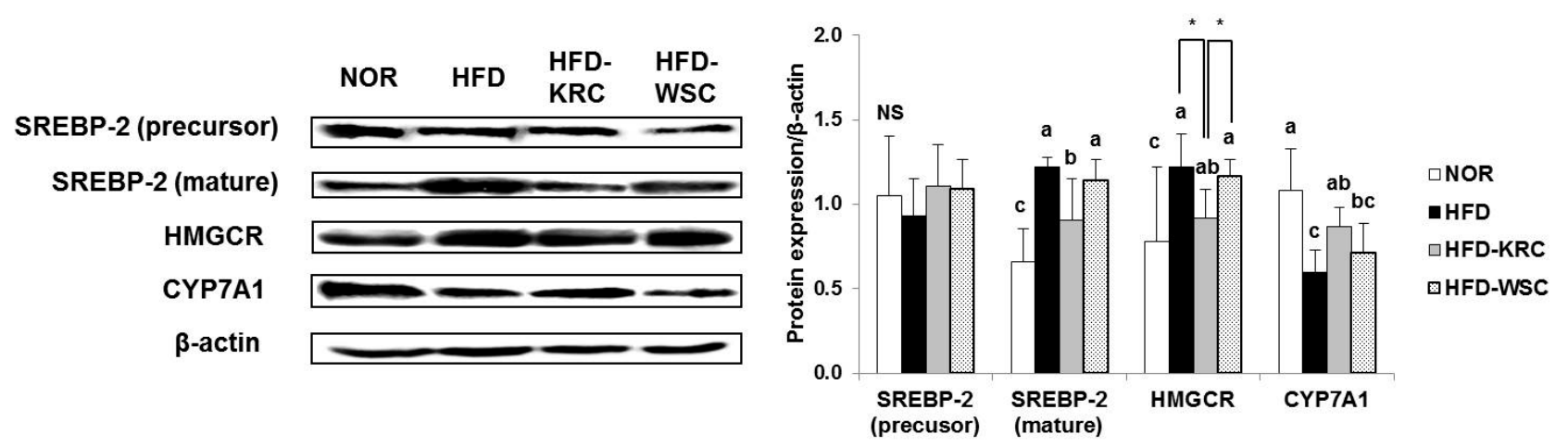

Figure 2. Protein expression of transcription factors and enzymes involved in cholesterol metabolism in the liver of mice fed a cookie-supplemented high-fat diet for 9 weeks. NOR, mice fed the AIN-76 diet; HFD, mice fed the high-fat diet only; HFD-KRC, mice fed the HFD supplemented with the Korean rice cookie; HFD-WSC, mice fed the HFD supplemented with the Western style cookie. ${ }^{a-c}$ Data with different letters are significantly different according to one-way ANOVA followed by Duncan's multiple range test at $\mathrm{p}<0.05$. "Significantly different according to Student's $t$-test at $\mathrm{p}<0.05$ between two groups. ${ }^{\text {NS }}$ Not significantly different at $\mathrm{p}<0.05$ 


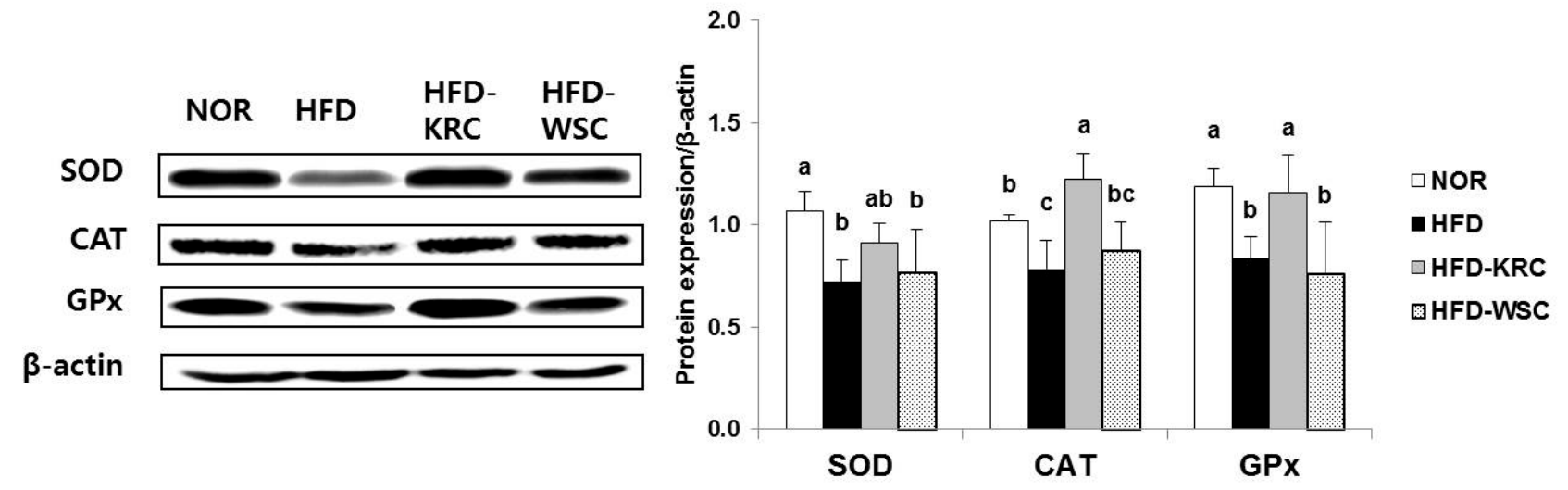

Figure 3. Protein expression of antioxidant enzymes in the liver of mice fed a cookie-supplemented high-fat diet for 9 weeks. NOR, mice fed the AIN76 diet; HFD, mice fed the high-fat diet only; HFD-KRC, mice fed the HFD supplemented with the Korean rice cookie; HFD-WSC; mice fed the HFD supplemented with the Western style cookie. ${ }^{\mathrm{a}-\mathrm{c}}$ Data with different letters are significantly different according to one-way ANOVA followed by Duncan's multiple range test at $\mathrm{p}<0.05$. CAT, catalase; GPx, glutathione peroxidase; SOD, superoxide dismutase

\subsection{Inhibition of Oxidative Stress by Korean Rice Cookie}

Hyperlipidemia is associated with increased oxidative damage [16] due to enhanced mitochondrial fatty acid oxidation [29]. In this study, the hepatic ROS, ONOO ${ }^{-}$, and TBARS levels were higher, and the GSH concentration was lower, in the HFD-fed group than in the NOR group $(p<0.05$, Table 3$)$. The concentrations of these oxidative stress-related markers in the HFD-WSC group were comparably high or even higher than those in the HFD group. However, the hepatic ROS and $\mathrm{ONOO}^{-}$concentrations of the HFD-KRC group were significantly decreased by $33.86 \%$ and $53.47 \%$, respectively, compared with the levels in the HFD-WSC group $(\mathrm{p}<0.05)$. The hepatic TBARS concentration of the HFD-KRC group was reduced by $40.13 \%$ compared with that of the HFD group $(p<0.05)$. On the other hand, the hepatic GSH level was higher in the HFD-KRC group than in the HFD and HFD-WSC groups (by 230\% and 246.43\%, respectively; $\mathrm{p}<0.05)$. Hyperlipidemia or hepatic lipid accumulation elevates oxidative stress. It has been reported that honey supplementation significantly reduced the MDA level and increases the GSH content in rat liver [30], the oxidative stress lowering effect of the KRC might in part be attributed to the antioxidant property of honey component.

\subsection{Elevation of the Antioxidant Status by Korean Rice Cookie}

Antioxidant enzymes constitute an organism's native defense system to eliminate free radicals, thereby preventing oxidative damage [31]. In the HFD group, the expression levels of the antioxidant enzymes superoxide dismutase (SOD), catalase (CAT), and glutathione peroxidase (GPx) were significantly decreased by $32.71 \%, 23.53 \%$, and $30.25 \%$, respectively, compared with those in the NOR group $(p<0.05$, Figure 3$)$. The levels of antioxidant enzyme expression in the HFD and HFD-WSC group were similar whereas those of CAT and GPx in the HFD-KRC group were significantly higher. Compare to the HFD-WSC group, CAT and GPx in the HFD-KRC were higher by $140.1 \% \quad(\mathrm{p}<0.05)$ and $152.24 \%$, respectively $(\mathrm{p}<0.05)$. It has been reported that honey supplementation restores the activities of CAT and GPx in the liver of young and middle-aged rats [32]. Moreover, oral supplementation of trichlorfon with pine tree honey, the activities of hepatic SOD, CAT, and GPX were increased in male BALB/C mice [33]. Therefore, the antioxidant effects of the $\mathrm{KRC}$ are very likely due to its honey component.

\section{Conclusion}

In this study, lipid-lowering and antioxidant effects were revealed in mice fed a HFD supplemented with KRC, dasik. These effects were greater than those for a traditional WSC. The rice cake flour, mung bean starch, and honey ingredients of the KRC were likely instrumental in decreasing the plasma and hepatic TG concentrations through the downregulation of TG synthesis and the upregulation of fatty acid oxidation. Moreover, the KRC augmented antioxidant enzyme expression in the liver of HFD-fed mice.

\section{Acknowledgements}

This research was supported by the Superiority and Functionality of Hansik (Korean Food) Research Program (No. 911040-1), iPET (Korea Institute of Planning and Evaluation for Technology in Food, Agriculture, Forestry and Fisheries), Ministry of Agriculture, Food and Rural Affairs.

\section{Statement of Competing Interests}

The authors have no competing interests.

\section{List of Abbreviations}

ACC, acetyl-CoA carboxylase; ACOX1, acyl-coenzyme A oxidase 1; ALT, alanine transaminase; AST, aspartate transaminase; CAT, catalase; CPT1, carnitine palmitoyltransferase 1; CYP7A1, microsomal cytochrome 
P450 family 7 subfamily A member 1; FAS, fatty acid synthase; GI, glycemic index; GPx, glutathione peroxidase; GSH, glutathione; HFD, high-fat diet; HMGCR, 3-hydroxy-3-methylglutaryl-CoA reductase; KRC, Korean traditional rice cookie; MDA, malondialdehyde; PBS, phosphate-buffered saline; PPAR- $\alpha$, peroxisome proliferator-activated receptor-alpha; ROS, reactive oxygen species; SOD, superoxide dismutase; SREBPs, sterol regulatory element-binding proteins; TBARS, thiobarbituric acid-related substances; TC, total cholesterol; TG, triglyceride; WSCs, western style cookies

\section{References}

[1] Piernas, C., Popkin, and B.M., "Trends in snacking among U.S. children”, Health affairs, 29 (3). 398-404. 2010.

[2] Karklina, D., Gedrovica, I., Reca, M., and Kronberga, M., "Production of biscuits with higher nutritional value", Proceedings of the Latvian Academy of Sciences. Section B. Natural, Exact, and Applied Sciences, 66 (3). 113-116. 2012.

[3] Bouchard-Mercier, A., Paradis, A.M., Godin, G., Lamarche, B., Perusse, L., and Vohl, M.C., "Associations between dietary patterns and LDL peak particle diameter: A cross-sectional study", Journal of the American College of Nutrition, 29 (6). 630-637. 2010.

[4] Choi, W.H., Gwon, S.Y., Ahn, J., Jung, C.H., and Ha, T.Y., "Cooked rice prevents hyperlipidemia in hamsters fed a highfat/cholesterol diet by the regulation of the expression of hepatic genes involved in lipid metabolism”, Nutrition Research, 33 (7). 572-579. 2013.

[5] Jenkins, D.J., Kendall, C.W., Augustin, L.S., Franceschi, S., Hamidi, M., Marchie, A., Jenkins, A.l., and Axelsen, M., "Glycemic index: overview of implications in health and disease", The American journal of clinical nutrition, 76 (1). 266S-273S. 2002.

[6] Parks, E.J., Skokan, L.E., Timlin, M.T., and Dingfelder, C.S., "Dietary sugars stimulate fatty acid synthesis in adults", The Journal of nutrition, 138 (6). 1039-1046. 2008.

[7] Yaghoobi, N., Al-Waili, N., Ghayour-Mobarhan, M., Parizadeh, S.M.R., Abasalti, Z., Yaghoobi, Z., Yaghoobi, F., Esmaeili, H., Kazemi-Bajestani, S.M.R., Aghasizadeh, R., Saloom, K.Y., and Ferns, G.A.A., "Natural honey and cardiovascular risk factors; effects on blood glucose, cholesterol, triacylglycerole, CRP, and body weight compared with sucrose”, The scientific world journal, 8. 463-469. 2008.

[8] Tang, J.J., Li, J.G., Qi, W., Qiu, W.W., Li, P.S., Li, B.L., and Song, B.L., "Inhibition of SREBP by a small molecule, betulin, improves hyperlipidemia and insulin resistance and reduces atherosclerotic plaques”, Cell metabolism, 13 (1). 44-56. 2011.

[9] Goldstein, J.L., DeBose-Boyd, R.A., and Brown, M.S., "Protein sensors for membrane sterols”, Cell, 124 (1). 35-46. 2006.

[10] Yang, J., Goldstein, J.L., Hammer, R.E., Moon, Y.A., Brown, M.S., and Horton, J.D., "Decreased lipid synthesis in livers of mice with disrupted Site-1 protease gene”, Proceedings of the National Academy of Sciences, 98 (24). 13607-13612. 2001.

[11] Ji, G., Zhao, X., Leng, L., Liu, P., and Jiang, Z., "Comparison of dietary control and atorvastatin on high fat diet induced hepatic steatosis and hyperlipidemia in rats", Lipids in health and disease, 10 (1). 23-10. 2011.

[12] Cao, Y., Bei, W., Hu, Y., Cao, L., Huang, L., Wang, L., Luo, D., Chen, Y., Yao, X., He, W., Liu, X., and Guo, J., "Hypocholesterolemia of Rhizoma Coptidis alkaloids is related to the bile acid by up-regulated CYP7A1 in hyperlipidemic rats", Phytomedicine, 19 (8). 686-692. 2012.

[13] Birben, E., Sahiner, U.M., Sackesen, C., Erzurum, S., and Kalayci, O., "Oxidative stress and antioxidant defense", World Allergy Organization Journal, 5 (1). 9-19. 2012.

[14] Willcox, J.K., Ash, S.L., and Catignani, G.L., "Antioxidants and prevention of chronic disease", Critical reviews in food science and nutrition, 44 (4). 275-295. 2004.
[15] Bowen, P.E. and Borthakur, G., "Postprandial lipid oxidation and cardiovascular disease risk”, Current atherosclerosis reports, 6 (6). 477-484. 2004.

[16] Bae, J.H., Bassenge, E., Kim, K.B., Kim, Y.N., Kim, K.S., Lee, H.J., Moon, K.C., Lee, M.S., Park, K.Y., and Schwemmer, M., "Postprandial hypertriglyceridemia impairs endothelial function by enhanced oxidant stress", Atherosclerosis, 155 (2). 517-523. 2001.

[17] Tsai, W.C., Li, Y.H., Lin, C.C., Chao, T.H., and Chen, J.H., "Effects of oxidative stress on endothelial function after a high-fat meal”, Clinical Science, 106 (3). 315-319. 2004.

[18] Folch, J., Lees, M., and Sloane-Stanley G.H., "A simple method for the isolation and purification of total lipids from animal tissues”, The Journal of Biological Chemistry, 226 (1). 497-509. 1957.

[19] Ohkawa, H., Ohishi, N., and Yagi, K.., "Assay for lipid peroxides in animal tissues by thiobarbituric acid reaction", Analytical biochemistry, 95 (2). 351-358. 1979.

[20] Ellman, M.A., "A colorimetric method for determining low concentrations of mercaptans", Archives of biochemistry and Biophysics, 74 (2). 443-450. 1958.

[21] Kooy, N.W., Royall, J.A., Ischiropoulos, H., and Beckman, J.S., "Peroxynitrite-mediated oxidation of dihydrorhodamine 123", Free Radical Biology and Medicine, 16 (2). 149-156. 1994.

[22] Jung, K., Hong, S.H., Kim, M., Han, J.S., Jang, M.S., and Song, Y.O., "Antiatherogenic effects of Korean cabbage kimchi with added short arm octopus”, Food Science and Biotechnology, 24 (1). 249-255. 2015.

[23] Longo, K.A., Wright, W.S., Kang, S., and Gerin, I., Chiang S.H., Lucas, P.C., Opp, M.R., and MacDougald, O.A., "Wnt10b inhibits development of white and brown adipose tissues", Journal of Biological Chemistry, 279 (34). 35503-35509. 2004.

[24] Elmquist, J.K., Coppari, R., Balthasar, N., Ichinose, M., and Lowell, B.B., "Identifying hypothalamic pathways controlling food intake, body weight, and glucose homeostasis", Journal of Comparative Neurology, 493 (1). 63-71. 2005.

[25] Ahima, R.S., "Digging deeper into obesity", The Journal of clinical investigation, 121 (6). 2076-2079. 2011.

[26] Yang, X.R., Wat, E., Wang, Y.P., Ko, C.H., Koon, C.M., Siu, W.S., Gao, S., Cheung, D.W.S., Lau, C.B.S., Ye, C.X., and Leung, P.C., "Effect of dietary cocoa tea (Camellia ptilophylla) supplementation on high-fat diet-induced obesity, hepatic steatosis, and hyperlipidemia in mice”, Evidence-Based Complementary and Alternative Medicine, 2013. 1-11. 2013.

[27] Al-Waili, N.S., "Natural honey lowers plasma glucose, C-reactive protein, homocysteine, and blood lipids in healthy, diabetic, and hyperlipidemic subjects: Comparison with dextrose and sucrose", Journal of medicinal food, 7 (1). 100-107. 2004.

[28] Parks, E.J., Skokan, L.E., Timlin, M.T., and Dingfelder, C.S., "Dietary sugars stimulate fatty acid synthesis in adults", The Journal of nutrition, 138 (6). 1039-1046. 2008.

[29] Pessayre, D., Mansouri, A., and Fromenty, B., "Nonalcoholic Steatosis and Steatohepatitis. V. Mitochondrial dysfunction in steatohepatitis", American Journal of Physiology-Gastrointestinal and Liver Physiology, 282 (2). G193-G199. 2002.

[30] Kilicoglu, B., Gencay, C., Kismet, K., Kilicoglu S.S., Erguder, I., Erel, S., Sunay, A.E., Erdemli, E., Durak, I., and Akkus, M.A., The ultrastructural research of liver in experimental obstructive jaundice and effect of honey. The American Journal of Surgery, 195 (2). 249-256. 2008.

[31] Wang, S.Y. and Gao, H., "Effect of chitosan-based edible coating on antioxidants, antioxidant enzyme system, and postharvest fruit quality of strawberries (Fragaria $\mathrm{x}$ aranassa Duch.)", LWT-Food Science and Technology, 52 (2). 71-79. 2013.

[32] Yao, L.K., Razak, S.L.A., Ismail, N., Fai, N.C., Asgar, M.H.A.M., Sharif, N.M., Aan, G.J., and Jubri, Z., "Malaysian gelam honey reduces oxidative damage and modulates antioxidant enzyme activities in young and middle aged rats", Journal of Medicinal Plants Research, 5 (23). 5618-5625. 2011.

[33] Petrus, K., Schwartz, H., and Sontag, G., Analysis of flavonoids in honey by HPLC coupled with coulometric electrode array detection and electrospray ionization mass spectrometry. Analytical and bioanalytical chemistry, 400 (8). 2555-2563. 2011. 